\title{
Measuring the hourly gain of the scintillator detectors from EAS data
}

\author{
V.B. Jhansi ${ }^{* a b}$, S. Ahmad ${ }^{a c}$, K.P. Arunbabu ${ }^{a b}$, A. Chandra ${ }^{a b}$, S.R. Dugad ${ }^{a b}$, S.K.

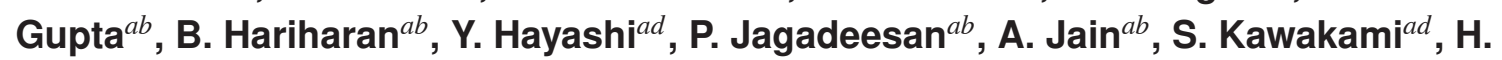 \\ Kojima $^{a e}$, P.K. Mohanty ${ }^{a b}$, S.D. Morris ${ }^{a b}$, P.K.Nayak ${ }^{a b}$, A. Oshima ${ }^{a f}$, B.S. Rao ${ }^{a b}$, L.V. \\ Reddy ${ }^{a b}$, S. Shibata ${ }^{a f}$, M.Zuberi $^{a b}$ \\ ${ }^{a}$ The GRAPES-3 Experiment, Cosmic Ray Laboratory, Raj Bhavan, Ooty 643001, India \\ ${ }^{b}$ Tata Institute of Fundamental Research, Mumbai 400005, India \\ ${ }^{c}$ Aligarh Muslim Univeristy, Aligarh 202002, India \\ ${ }^{d}$ Graduate School of Science, Osaka City University, Osaka 558-8585, Japan \\ ${ }^{e}$ Faculty of Engineering, Aichi Insitute of Technology, Toyota City, Aichi 470-0392, Japan \\ ${ }^{f}$ College of Engineering, Chubu University, Kasugai, Aichi 487-8501, Japan
}

E-mail: jhansi1990@gmail.com

\begin{abstract}
The GRAPES-3 experiment is an array of $\sim 400$ plastic scintillator detectors with a compact configuration at Ooty in India. An accurate measurement of the gain of each detector is an important requirement for accurate determination of the energy of primary cosmic rays. The old method used earlier relied on manual placement of muon paddles for determining the signal produced by single muons in the detector. However, the number of detectors being rather large, it takes around 40 days to completete one round of calibration. Therefore each detector gets calibrated only 6-8 times in a year. However, the gain of detectors may vary significantly over this period. Also these detectors display gain variations due to changes in temperature. Since the estimated particle densities in an EAS depend on detector gains, their infrequent measurement may lead to incorrect estimate of shower size. In new method the gains are calculated on an hourly basis from the EAS data, which avoids the problems listed above. Details of this method will be presented at the conference.
\end{abstract}




\section{GRAPES-3 Array}

The GRAPES-3 EAS array consists of $\sim 400$ scintillator detectors arranged in the form of hexagonal rings with only $8 \mathrm{~m}$ separation between adjacent detectors. The detectors used in GRAPES-3 are of two distinct types, the cone and the fiber detectors, which have a different geometry and method of collection of scintillation photons. Each detector consists of four scintillator slabs, each of which has an area of $50 \times 50 \mathrm{~cm}^{2}$. The scintillators used in cone detectors are $5 \mathrm{~cm}$ thick and those in fiber detectors are $2 \mathrm{~cm}$ thick. The array is located at Ooty $\left(11.4^{0} \mathrm{~N}\right.$ latitude, $76.7^{0} \mathrm{E}$ longitude and $2200 \mathrm{~m}$ altitude) at an atmospheric depth of $800 \mathrm{gm} / \mathrm{cm}^{2}$.

In a cone detector, a $5 \mathrm{~cm}$ diameter photomultiplier tube (PMT) model ETL9807B is mounted on top of a trapezoidal shaped aluminium cone with its photocathode at a height of $60 \mathrm{~cm}$ above the scintillator surface, as shown in Figure 1a. The inner surfaces of the tank and the cone are painted with super-white $\left(\mathrm{TiO}_{2}\right)$ paint to increase the efficiency of collection of diffuse photons at the PMT. A small fraction of emitted photons produced by the passage of an ionizing charged particle in the scintillator is guided to the PMT by multiple reflections from the inner surface of the aluminium container. Subsequently, the PMT converts the photons into an electrical pulse with a typical rise time of $3 \mathrm{~ns}$ and an amplitude of a few $\mathrm{mV}$. The whole detector assembly is covered by a second alu minium cone to protect it from rain and heating due to direct sunlight. However, in a fiber detector, which is shown in Figure 1b, photons are collected at the PMT by using wavelength shifting fibres which are coupled to the scintillator surface. The electrical pulse from the PMT is fed into the corresponding ADC channel which then registers the amount of charge delivered by the signal [1] [2].

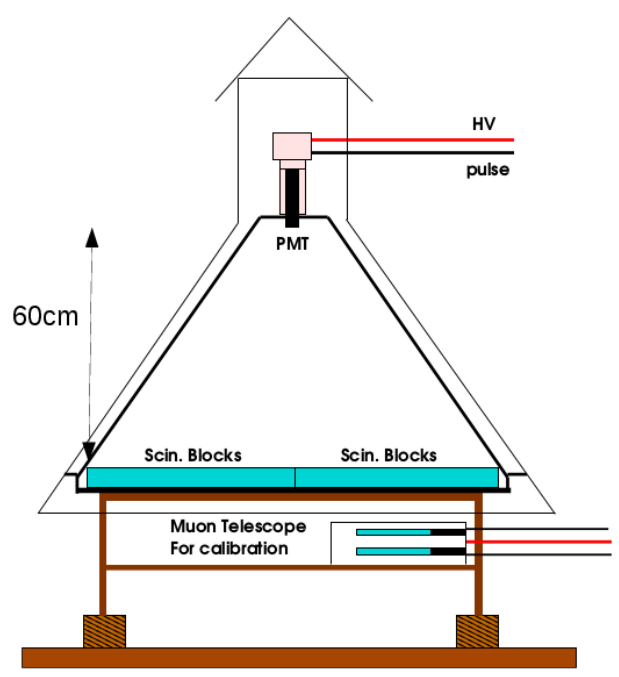

(a) A Cone detector

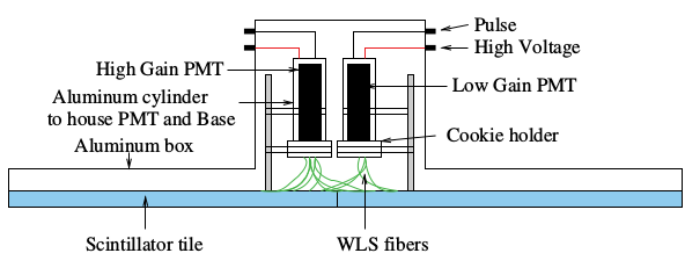

(b) A Fiber detector

Figure 1: Types of detectors

\section{The conventional method for calculation of gain}

An accurate measurement of gain of these detectors is very crucial for the determination of 
shower size. The conventional method employed is to measure the integrated charge of the signals produced by single muons .For this purpose, each detector is calibrated using a small muon telescope (also called paddle) assembled from two independent scintillators $(2 \mathrm{~cm}$ thick) placed inside an aluminium box with a vertical separation of $10 \mathrm{~cm}$. To calibrate a detector the paddle is kept below the detector as shown in Figure 1a [1]. The two scintillators inside the paddle are connected separately to two independent discriminators which produce a pulse of width $150 \mathrm{~ns}$. The output of each discriminator is then fed into a coincidence unit. When a muon passes through both scintillators in the paddle, the coincidence unit produces a GATE pulse of width $620 \mathrm{~ns}$. This GATE pulse serves as the calibration trigger. The calibration trigger enables the amount of energy deposited by a single muon to be recorded by the ADC. The integrated charge contained in the signal is proportional to the amount of energy deposited by a single muon in the scintillator detector.In GRAPES-3, charge integrating ADCs. While the calibration paddle is kept below the detector as shown in the Figure 1a, the ADC channels are used to record the spectrum of pulses produced by single muons. The ADC distribution of detector 54 for 4 th May 2014, for run numbers 65200-65461 is shown in Figure 2.

Even in the absence of a signal at the ADC input, the ADC integrates a fixed portion of the GATE signal and registers that value for each ADC channel. This ADC value, called the pedestal, is visible as a sharp peak in the integrated charge distribution shown in Figure 2. The charge deposited during the passage of muons is recorded and its distribution is seen as the second peak in Figure 2. The gain of a scintillator is defined as the difference between the peaks of the two distributions as shown in Figure 2.

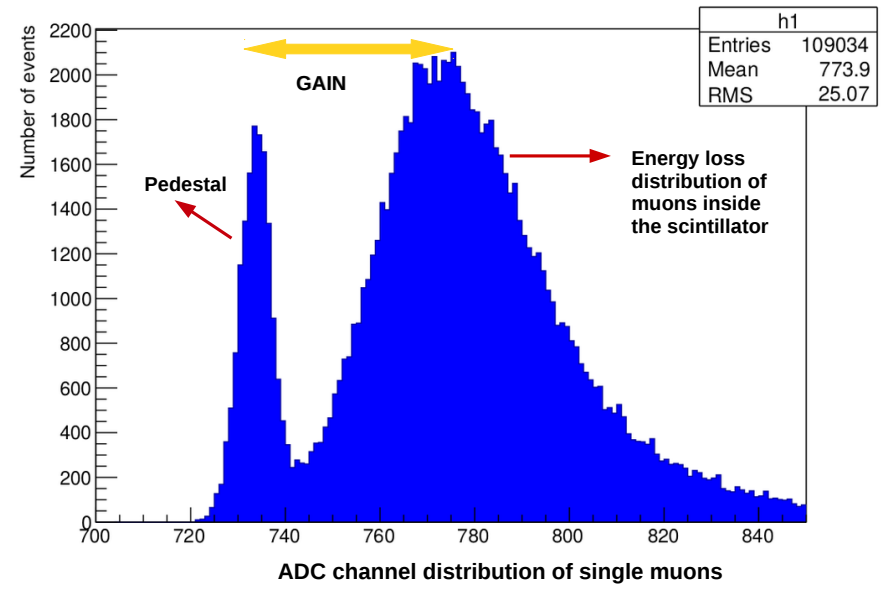

Figure 2: ADC distribution of single muons

The value of gain varies from detector to detector, depending on the scintillation efficiency and geometry of the detector. The gain of a given detector can also vary with time due to various factors such as the PMT, the high voltage, cables and electronics. For this reason each detector is calibrated 5-6 times in a year. (It takes approximately 2 months to complete one round of calibration for all the detectors). However the gain of the detector can vary significantly even during the intermittent period. In addition, the photocathode and dynode sensitivity of PMT vary periodically 
with temperature which is depicted in Figure-3. Hence we devised a new technique to determine the gain of the detector from the shower data.

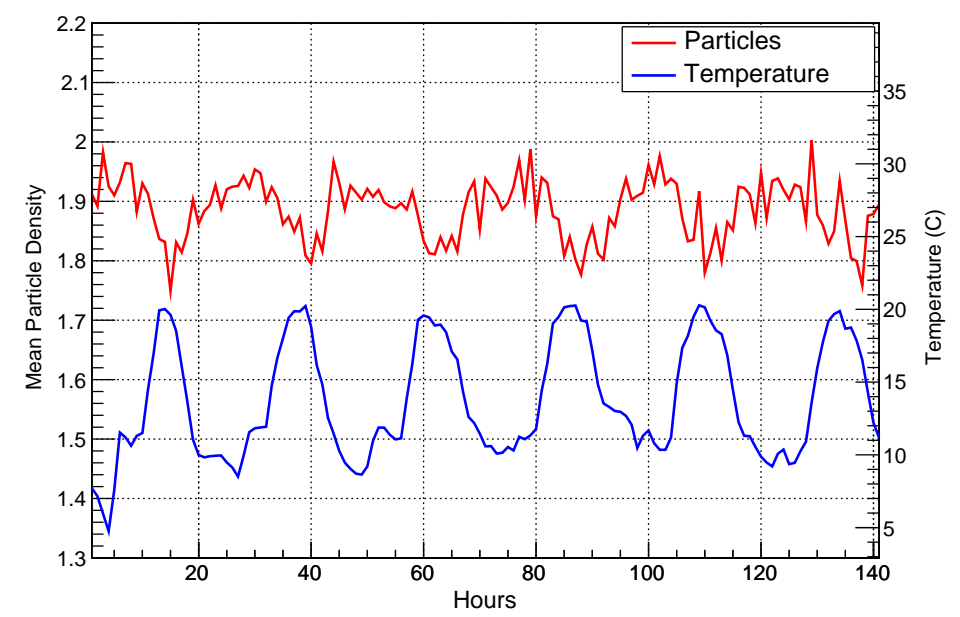

Figure 3: Variation of mean number of particles with temperature for Detector No:1

\section{Determination of gain from EAS data}

During calibration, which extends typically over a period of one hour, both signals produced by shower particles(single/multiparticles) and single muons are registered in ADC. The trigger system enables us to distinguish between the signals produced by single mouns and shower particles. As mentioned previously, signal produced by a single muon is recorded only when the ADC recieves the calibration trigger. Whereas, the trigger for recording signals produced by shower particles,consists of two levels namely Level-1 and Level-0. (The steps involved in generating a Level-0 trigger are as follows. The signals from all detectors in a given line (along the North-South direction) are OR'ed together. Then a $100 \mathrm{~ns}$ wide 3 -fold coincidence is generated among the line OR's for each combination of three successive lines. All these coincidences are then OR'ed together to generate the Level-0 trigger. Only the inner 9 rings have been used to generate this trigger. A Level-1 trigger is generated only when a minimum of 14 out of the 234 detectors, which participate in the trigger [1].The triggers thus generated act as GATE inputs to the ADCs. Each event recorded in the ADC is tagged by the type of trigger that produced it.

Initially, we will utilize the shower data recorded during calibration, which typically extends over one hour. During this period, the gain of the detector is known accurately,and the median number of particles interacting with the detector is calculated by taking the ratio of the median of net charge deposited in the ADC to the gain. The same quantity is calculated during each calibration and it is expected to change with time only within a few percentage. However it may vary from detector to detector depending on its position and efficiency.

Since each detector is calibrated 5-6 times in a year,we get those many measurements of true value of median number of particles. The measurements taken for two detectors,cone and fiber each, are shown in Figure4.The mean of these measurements for each detector is used for further 
analysis.

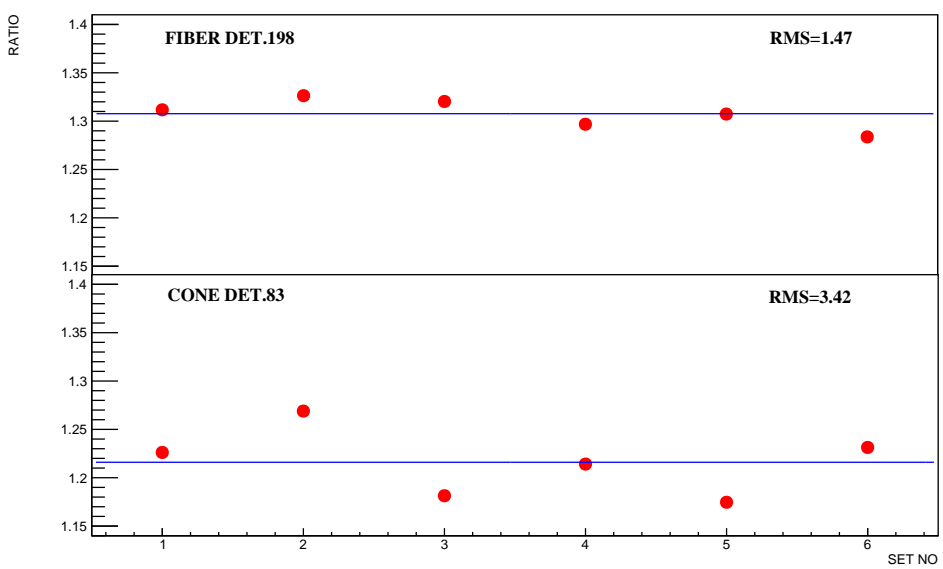

Figure 4: Median number of particles

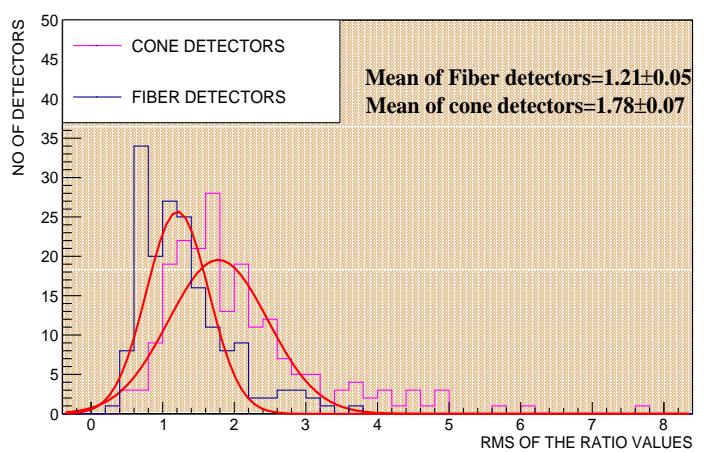

(a) Median number of particles

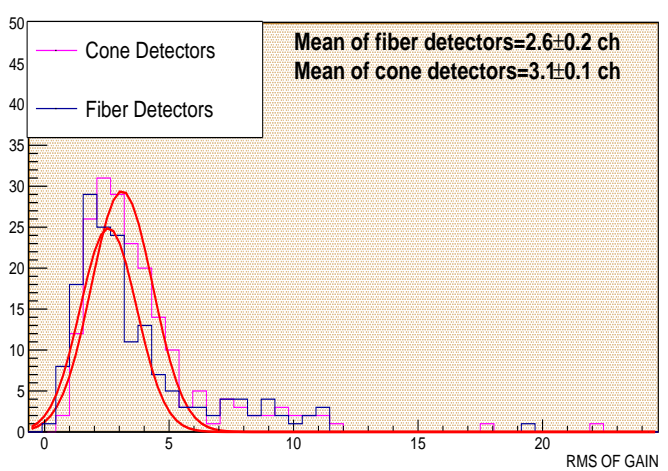

(b) Gain

Figure 5: Distribution of RMS

It is observed that the RMS is larger for the cone detector.In addition,the distribution of RMS for all the 378 detectors is shown in Figure5a. .It is observed that distribution for cone detectors is slightly shifted towards the right, and the mean RMS for fiber and cone detectors are $1.21 \pm 0.05 \%$ and $1.78 \pm 0.07 \%$ respectively. The larger RMS for cone detectors is due to non uniform collection of photons by the PMT. In case of cone detectors the PMT is kept at a certain height above the scintillators. As a result, the number of the photons collected by the PMT depends on the position of passage of charged particles through the scintillator. For example, more photons reach the PMT if charged particle passesthrough the centre of the detector, rather than its corner. This produces a gain variation of about $30 \%$ from the centre to the corner of the detector. Hence the gain measured is highly dependent on the position of placement of paddles beneath the detector. A slight misplacement of paddles can lead to miscalculation of gain. However in case of fiber detectors, the photons are collected by wavelength shifting fibers and hence the non-uniformity is only about $7 \%$. 
Each detector gets triggered by about 1 lakh showers in a day. By calculating the median amount of charge deposited in ADC, by all the showers, it is possible to calculate the gain(computed gain) on daily basis by normalising its value to the true value(which is calculated previously). The variation of computed gain on daily basis for Detector No:198 over a span of one year is shown in Figure6. The gain obtained from calibration is also shown in the same figure.

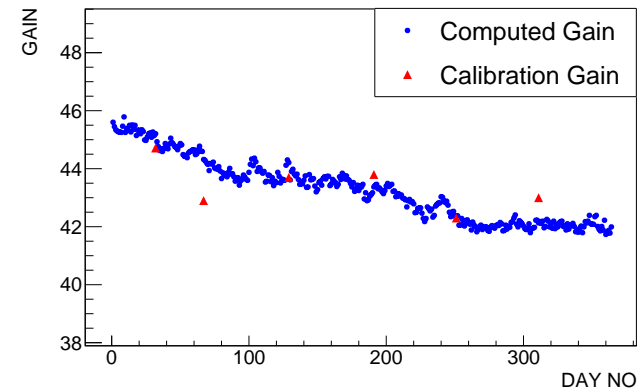

(a) Variation of daily gain

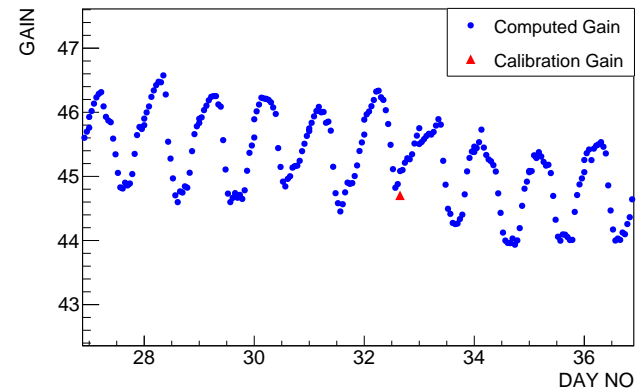

(b) Variation of gain with temperature

Figure 6: Computed gain

As mentioned previously, the gain of the detector varies with photocathode and dynode efficiency. The average temperature coefficient for total number of particles normalised over trigger rate is $-0.31 \% / \mathrm{C}$. The gain is further corrected for changes due to temperature. The temperature corrected gain of detector No:198 for a period of few days is shown in Figure 6.

Further on, the calibration gain is compared with the computed gain calculated during the same period when calibration is done. The difference between both the gains(in \%) for detector NO:198 is plotted in Figure 7. Evidently, the RMS has reduced from 1.9 ch to $1.1 \mathrm{ch}$ after temperature correction.

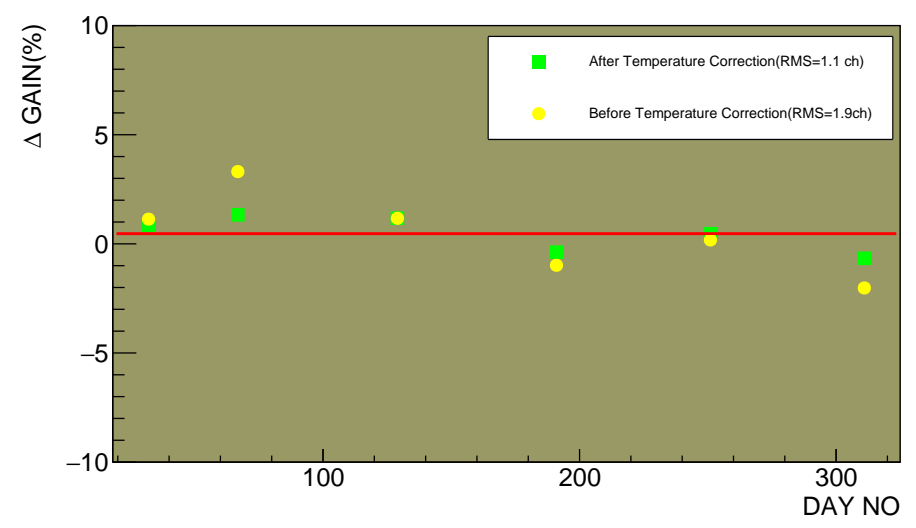

Figure 7: Daily variation of gain

\section{4. conclusion}

An accurate measurement of gain is essential for the accurate determination of energy of pri- 
mary cosmic rays.Earlier, using the old method,we could not measure gain frequently. However the GRAPES-3 experiment, which has a trigger rate of $35 \mathrm{~Hz}$,about 3 lakh showers are registered in a day per detector, and we have utilised the these EAS particles instead of single muons for a more accurate measurement of gain. The gain ,thus calculated, is accurate to $1.5 \%$. Hence it is a major improvement over the existing method.

\section{References}

[1] S.K. Gupta et al, GRAPES-3-A high density air-shower array for studies on the structure on the cosmic ray energy spectrum near the knee, Nuclear Instruments and Methods in Physical Research A 540(2005) 311-323

[2] P.K. Mohanty et al, Measurement of some EAS properties using new scintillator detectors developed for the GRAPES-3 experiment Astroparticle Physics 31 (2009)24-36 